\title{
Log-Normal Turbulence Dissipation in Global Ocean Models
}

\author{
Brodie Pearson and Baylor Fox-Kemper \\ Department of Earth, Environmental and Planetary Sciences, Brown University, Providence, Rhode Island 02906, USA
}

(Received 25 October 2017; published 26 February 2018)

\begin{abstract}
Data from turbulent numerical simulations of the global ocean demonstrate that the dissipation of kinetic energy obeys a nearly log-normal distribution even at large horizontal scales $\mathcal{O}(10 \mathrm{~km})$. As the horizontal scales of resolved turbulence are larger than the ocean is deep, the Kolmogorov-Yaglom theory for intermittency in 3D homogeneous, isotropic turbulence cannot apply; instead, the down-scale potential enstrophy cascade of quasigeostrophic turbulence should. Yet, energy dissipation obeys approximate lognormality—robustly across depths, seasons, regions, and subgrid schemes. The distribution parameters, skewness and kurtosis, show small systematic departures from log-normality with depth and subgrid friction schemes. Log-normality suggests that a few high-dissipation locations dominate the integrated energy and enstrophy budgets, which should be taken into account when making inferences from simplified models and inferring global energy budgets from sparse observations.
\end{abstract}

DOI: 10.1103/PhysRevLett.120.094501

The mechanical energy budget of the oceans must close independently because of the minimal compressibility of seawater [1]. The global oceans accumulate potential and kinetic energy (KE) mostly from the winds and tides at large horizontal scales, which flow to concentrate in boundary currents and turbulent flows. KE is ultimately dissipated near the Kolmogorov microscale, $\lambda_{K} \approx \mathcal{O}(1 \mathrm{~mm})$. The present highest-resolution global ocean models (GOMs) can now resolve some turbulence in the ocean [here nominal $0.1^{\circ}=\mathcal{O}(10 \mathrm{~km})$ horizontal and $\mathcal{O}(10-100 \mathrm{~m})$ vertical resolution], but the direct representation of the $\lambda_{K}$-scale KE sinks remains centuries away, and instead present models use bulk formulas or "subgrid schemes," which dissipate KE in the boundary layers and ocean interior and represent the transfer of $\mathrm{KE}$ to unresolved scales [2]. This Letter explores the statistics of dissipation in GOMs which resolve the ubiquitous quasigeostrophic (QG) turbulence of the ocean but do not resolve $\lambda_{K}$. Because of the limited resolution of GOMs, these statistics provide information about the intermittency of QG turbulence rather than the $\lambda_{K^{-}}$ scale dissipation that, in the real world, could occur in a different manner and location (e.g., $[3,4])$.

QG turbulence [5] is an asymptotic theory for strongly nonlinear flow in stratified, rotating fluids (such as the ocean) in the "mesoscale" near the 5-300 km Rossby deformation scale $l_{R}$, where turbulence is constrained to nearly horizontal motions [6]. Oceanic baroclinic and

Published by the American Physical Society under the terms of the Creative Commons Attribution 4.0 International license. Further distribution of this work must maintain attribution to the author(s) and the published article's title, journal citation, and DOI. barotropic instabilities set the energy injection scale near the Rossby radius $\left(l_{\text {inj }} \approx l_{R}\right)$, and the model presented here resolves this scale as its horizontal resolution $\Delta_{h} \ll l_{R}$ at most latitudes [7]. QG turbulence resembles two-dimensional (2D) turbulence but includes density stratification, weak vertical motion, and planetary rotation. QG, like 2D, turbulence develops two cascades of conserved invariants: an inverse (up-scale) transfer of KE toward larger length scales for $l>l_{\text {inj }}$ and a forward (down-scale) flux of enstrophy (squared vorticity)—specifically, quasigeostrophic potential enstrophy (QGPE) - for $l<l_{\text {inj }}[8,9]$. Up-scale KE and down-scale QGPE cascades distinguish QG turbulence from 3D isotropic turbulence, which has only a down-scale KE cascade [10,11]. Despite QG predictions, numerical simulations of the ocean often show a down-scale KE cascade [12-14] to the "submesoscales" (non-QG turbulence much smaller than $l_{R}$ ), and submesoscales have been observed to receive $\mathrm{KE}$ from larger scales [15]. However, these studies used limited observations in one region or highly idealized model setups. Here the intermittency of the KE cascade of turbulence is analyzed in global, realistic numerical simulations of the hydrostatic Boussinesq equations. The present GOM is typically consistent with, but more general than, the QG theory; however, it produces a KE cascade to unresolved scales, and its cost requires sacrificing the resolution of most submesoscale motions [16]. We shall discuss the link between the KE cascade seen in simulations and the inertial QGPE cascade of the QG theory, as well as their respective intermittency statistics.

GOMs use subgrid schemes that mimic horizontal friction to dissipate $\mathrm{KE}$ and enstrophy, which estimates the forward KE transfer by QG turbulence to unresolved scales. Not all numerical and subgrid schemes precisely 
relate the rate of production, rate of cascade, and rate of dissipation to one another. In the present study, we restrict our analyses to dissipation statistics, and we use a variety of subgrid schemes to ensure robust conclusions. While it is difficult in practice to vary resolution in a realistic GOM, our previous study [17] examines resolution convergence in an idealized setting with the same schemes and predicts that the mesoscale turbulence here would be unaffected by quadrupling the resolution over most of the Earth [7], consistent with the agreement between GOMs at this resolution and observations [18]. Dissipation is one of the largest sinks of KE in GOMs, rivaling topographic drag and boundary layer turbulence [19]. It is therefore important to quantify the spatial intermittency of dissipation so that the energy transfer to, and effects of, unresolved turbulence can be approximated in a way that is physically consistent with this intermittency.

The energy cascade in 3D turbulence is well known to be intermittent [20-22]. The energy and enstrophy cascades in 2D turbulence are likewise intermittent [23-26] with some explored mechanisms [27,28]. In this Letter, we investigate the intermittency of dissipation in global ocean models which resolve QG turbulence.

Equations.-The evolution of the horizontal velocity $\mathbf{u}=(u, v, 0)$ in GOMs is a function of pressure $p$, planetary vertical vorticity $\mathbf{f}$, and vertical $\mathbf{F}_{\mathbf{V}}$ and horizontal $\mathbf{F}_{\mathbf{H}}$ friction given by

$$
\frac{D \mathbf{u}}{D t}=-\boldsymbol{\nabla} p+\mathbf{f} \times \mathbf{u}+\mathbf{F}_{\mathbf{V}}+\mathbf{F}_{\mathbf{H}},
$$

where $D / D t$ is the material derivative and $\boldsymbol{\nabla}=\left(\partial_{x}, \partial_{y}, 0\right)$. The hydrostatic relationship and incompressibility provide vertical velocity:

$$
\frac{\partial p}{\partial z}=-\rho g, \quad \boldsymbol{\nabla} \cdot \mathbf{u}+\frac{\partial w}{\partial z}=0 .
$$

This Letter focuses on $\mathbf{F}_{\mathbf{H}}$, which represents the mixing effects of unresolved mesoscale (QG) turbulence. A common parameterization for $\mathbf{F}_{\mathbf{H}}$ is a horizontal, harmonic "eddy" viscosity acting on the resolved velocity. $\mathbf{F}_{\mathbf{V}}$ is dominated by the effects of other processes, such as internal waves or boundary layer turbulence, and will not be considered further. Contracting (1) with $\mathbf{u}$, the effect of $\mathbf{F}_{\mathbf{H}}$ on $\mathrm{KE}$ is

$$
\mathbf{u} \cdot \mathbf{F}_{\mathbf{H}}=\mathbf{u} \cdot(\boldsymbol{\nabla} \cdot[\nu \boldsymbol{\nabla} \mathbf{u}])=\boldsymbol{\nabla} \cdot \mathbf{T}-\nu \frac{\partial u_{i}}{\partial x_{j}} \frac{\partial u_{i}}{\partial x_{j}},
$$

where $\nu$ is a dynamic viscosity depending on the flow and resolution parameters and repeated indices imply a summation over horizontal directions. $\mathbf{T}$ transports and diffuses $\mathrm{KE}$, but $\boldsymbol{\nabla} \cdot \mathbf{T}$ integrates to zero over the global ocean. The last subtracted term is the dissipation $\varepsilon$. Dissipation is positive definite if $\nu>0$. Dissipation estimates for nonharmonic friction are found similarly [19]. Hydrostasy and the grid aspect ratio $[\delta=\mathcal{O}(0.05-0.005)]$ imply that the vertical KE is negligible: $w w /(\mathbf{u} \cdot \mathbf{u})=\mathcal{O}\left(\delta^{2}\right)$.

This study uses the high-resolution Parallel Ocean Program (POP) numerical model, described in Refs. [18,19,29], which has $\Delta_{h} \ll l_{\text {inj }}$ everywhere except high latitudes and boundaries [7]. For most simulations shown here, $\nu$ is parameterized by the "2D Leith" large-eddy simulation technique [17,30], which avoids overdamping of the resolved flow [19]. However, qualitatively similar results occur with other parameterizations (see below). Most results shown use snapshots of the flow field on January 1 after a multiyear spin-up using realistic atmospheric forcing.

Results.-The global histogram for the logarithm of dissipation is shown in Fig. 1 at several depths. The most likely dissipation value decreases with the depth, consistent with the greatest velocities being near the surface. The PDFs of $\log _{10}(\varepsilon)$ are approximated well by a normal distribution, with the best-fit Gaussian curves shown. Deviations from log-normality are small but depth dependent. For example, skewness is slightly negative near the surface and slightly positive at a depth.

A theory predicting the log-normality of dissipation of QGPE, enstrophy, and KE is briefly sketched here and connects the distribution of the QGPE flux $\eta$ from large to small scales, following arguments similar to Ref. [20] for the 3D turbulent cascade of KE, with flux $\epsilon$. The direct evaluation of $\eta$ over a volume, eddy, or structure is possible, in principle [8,17], but, as with the diagnosis of $\epsilon$, the statistics of the dissipation rate of QGPE are more straightforward given the geographic and numerical complexities in this model. Suppose the eddy with the largest volume, at the top of a QGPE cascade, fluxes at a rate $\eta_{0}$, so that QGPE is transferred into smaller "child" eddies volumetrically dividing the original. There are more child

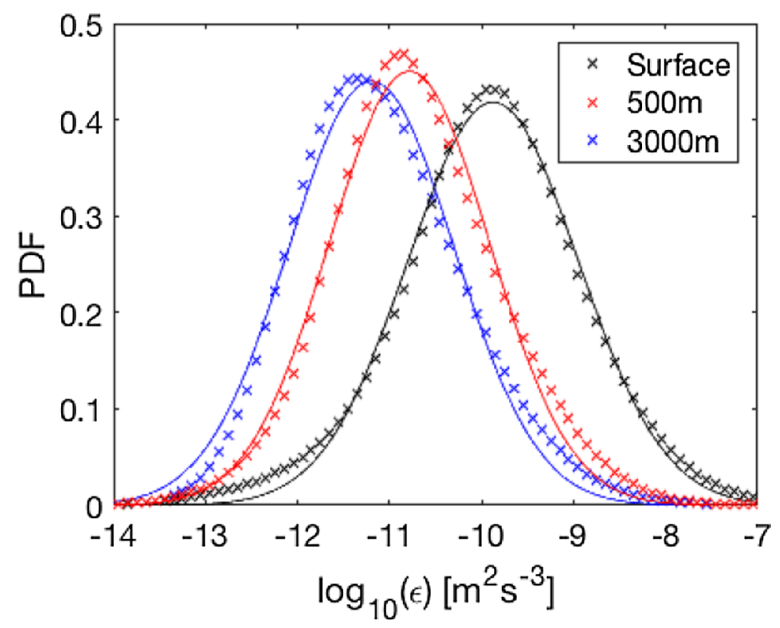

FIG. 1. Probability distribution functions (PDFs) for the logarithm of dissipation (symbols), and for the best-fit Gaussian function for each set of dissipation values (curves). The dissipation PDFs are shown for the surface (black), $500 \mathrm{~m}$ depth (red), and $3000 \mathrm{~m}$ depth (blue). 
eddies, each of which takes on a (non-negative) fraction $\beta$ of the QGPE flux arriving from the largest eddy $\eta_{0}$. For example, if the child eddies are $1 / 8$ as large as the original eddy, then on average each fills one of eight smaller volumes and fluxes $\bar{\beta}_{1} \eta_{0}=1 / 8 \eta_{0}$. If each child eddy is composed of eight smaller grandchildren, they each flux on average $1 / 8$ of whatever fraction of $\eta_{0}$ their parents flux, or $\bar{\beta}_{2} \bar{\beta}_{1} \eta_{0}=1 / 8 \times 1 / 8 \eta_{0}$. The geometric details and scale of each refinement and whether there are eight or another number of children are unimportant. The important factors for log-normality across averages of $\eta$ over equal volumes are that this number is constant over all cascade steps, that the child volumes do not intersect, and that the union of all child volumes equals the parent volume at each step. Relaxing these assumptions may result in multifractals rather than log-normality [31]. Any particular eddy will deviate from the average $\bar{\beta}_{i}$, so let $\beta_{i}$ represent its proportion $i$ out of $n$ steps down the cascade from the largest eddy. As the transfer continues, $n$ grows, so if the QGPE cascade is sufficiently deep and statistically stationary, then the large- $n$ statistics of $\beta_{i}$ will also govern the QGPE dissipation. The QGPE flux through any particular eddy $\left(\eta_{n}\right)$ can be found from the product of the proportions of fluxes at each larger scale up to the largest eddy; thus,

$$
\eta_{n}=\eta_{0} \prod_{i=1}^{n} \beta_{i}, \quad \log \left(\eta_{n} / \eta_{0}\right)=\sum_{i=1}^{n} \log \left(\beta_{i}\right) .
$$

From this construction and the assumed self-similarity, we assume the $\log \beta_{i}$ are independent and identically distributed with a finite mean and variance; thus, the summation in Eq. (4) is normally distributed for large $n$. The logarithm of the dissipation of QGPE should balance the fluxes and is thus normally distributed (histogram not shown, but skewness and kurtosis shown in Fig. 3).

Figure 1 shows that the dissipation of $\mathrm{KE}$ is log-normally distributed like the QGPE dissipation in Eq. (4). Figure 2 shows that averages over a variety of scales each share the log-normal character, consistent with the preceding arguments. The dissipation of 2D enstrophy $\left(\approx \nu\left|\nabla q_{2 \mathrm{D}}\right|^{2}\right)$ and QGPE $\left(\approx \nu\left|\nabla q_{\mathrm{QG}}\right|^{2}\right)$ are also approximately log-normal in the simulations, where $q_{2 \mathrm{D}}$ and $q_{\mathrm{QG}}$ are the vertical vorticity and QG potential vorticity, respectively, with gradients

$$
\begin{gathered}
\boldsymbol{\nabla} q_{2 \mathrm{D}}=\boldsymbol{\nabla}(\hat{\mathbf{z}} \cdot \boldsymbol{\nabla} \times \mathbf{u}), \\
\nabla q_{\mathrm{QG}}=\boldsymbol{\nabla} q_{2 \mathrm{D}}+\frac{\partial}{\partial z} \frac{f}{N^{2}} \nabla b .
\end{gathered}
$$

Figure 3 shows the QGPE dissipation statistics (gray lines), which are more log-normal than the KE dissipation statistics (black lines), for one of the simulations. The 2D enstrophy dissipation statistics (not shown) are similar to the QGPE dissipation statistics. Again gradients act over horizontal directions only, $f$ is the Coriolis parameter, $N$ is the buoyancy frequency, and $b$ is the buoyancy. Bachman, Fox-Kemper, and Pearson [17] detail the derivation of these dissipation operators.

The argument above explains why the dissipation of QGPE is nearly log-normal but not why those of KE and enstrophy are. At a scale $L$ much smaller than $l_{\text {inj }}$, the QGPE is increasingly dominated by the 2D enstrophy, as the ratio of the contribution to $q_{\mathrm{QG}}$ of the second term (vortex stretching) to the first term (vertical vorticity, $q_{2 \mathrm{D}}$ ) in (6) decreases as $L^{2} / l_{\text {inj }}^{2}$ [17]. Thus, if $L \ll l_{\text {inj }}$, then $q_{\mathrm{QG}} \approx q_{2 \mathrm{D}}$, and $2 \mathrm{D}$ enstrophy dissipation will be lognormal. Finally, in rotating, stratified turbulence, the Helmholtz decomposition of horizontal velocity is expected to be dominated by the rotational rather than the divergent velocity [32]. Thus, wherever $2 \mathrm{D}$ enstrophy is dissipated, then KE dissipation must cooccur with a similar statistical distribution. QGPE flux is conserved over all scales smaller than $l_{\text {inj }}$, so the QGPE flux and dissipation should be approximately equal. However, neither 2D enstrophy nor KE fluxes are guaranteed to be conserved across the scales of the QGPE cascade. Indeed, the QG theory predicts that KE dissipation should become increasingly small on scales smaller than $l_{\text {inj }}$. Bottom drag, vortex tilting, vertical viscosity, internal waves, and inverse cascades set quite different dynamics over these scales than a forward cascade for 2D enstrophy and KE. Observations in the North Atlantic show that, on submesoscales, waves begin to dominate the KE spectrum, changing the Helmholtz decomposition away from its turbulent scaling [33]. Only a
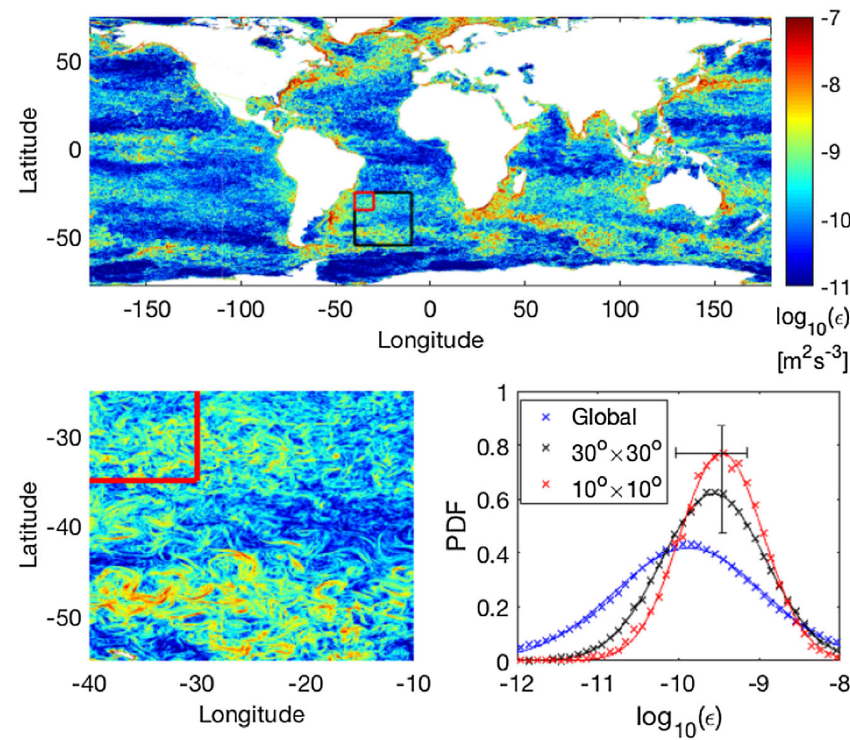

FIG. 2. (Top) Map of surface dissipation. (Bottom left) Surface dissipation map for a $30^{\circ} \times 30^{\circ}$ region denoted by a black box. (Bottom right) PDFs of $\log _{10}(\varepsilon)$, as in Fig. 1, for the global ocean (blue), the $30^{\circ} \times 30^{\circ}$ region (black), and the $10^{\circ} \times 10^{\circ}$ region (red). Also shown are the range of the mean and amplitude of Gaussian fits for the nine $10^{\circ} \times 10^{\circ}$ child regions within the black box [illustrating $\log \left(\eta_{n}\right)$ distributions from (4)]. 


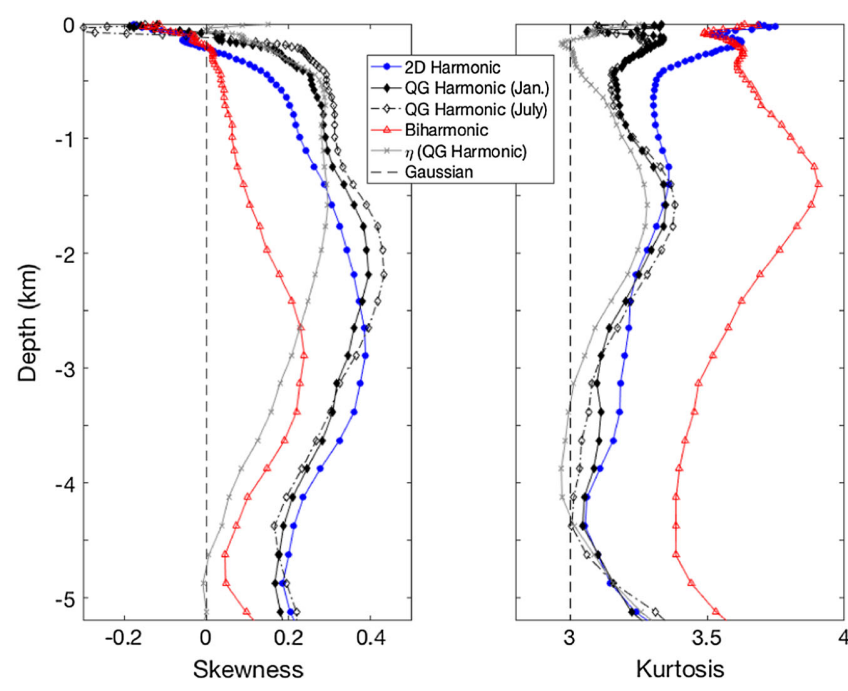

FIG. 3. Skewness (left) and kurtosis (right) of the global $\log _{10}(\varepsilon)$ PDF as a function of the depth for several simulations. For the QG harmonic simulation, the $\log _{10}(\varepsilon)$ statistics for both January and July are shown, as well as the statistics of the QGPE dissipation $\left[\log _{10}(\eta)\right.$; gray crosses]. The statistics are consistently near the Gaussian values.

restrictive conclusion is intended here: Log-normal dissipation of QGPE by horizontal friction implies log-normal dissipation of 2D enstrophy and log-normal dissipation of $\mathrm{KE}$ by horizontal friction when the grid is finer than $l_{\text {inj }}$ and $\mathrm{KE}$ and $q_{2 \mathrm{D}}$ are dominated by the turbulent, rotational velocity. Nonetheless, Figs. 1-3 show that models of the mesoscale and large submesoscales obey this limit.

Figure 2 shows global and regional maps of surface dissipation. High spatial variability is consistent with the log-normal distribution, but regions of dissipation are also related to oceanic features: The largest dissipation occurs in western boundary currents and the Antarctic Circumpolar Current. The PDFs of $\log _{10}(\varepsilon)$ for the global ocean and two smaller regions denoted by the black and red boxes on the global map are inset in Fig. 2. The dissipation is log-normal in each region (and all others examined), but the mean and variance depend upon the size and location of the chosen region. Interestingly, the amplitude and mean do not vary monotonically with the region size.

Deviations from log-normality are assessed through moments of the $\log _{10}(\varepsilon)$ distribution (Fig. 3, blue curve). The skewness and kurtosis are close to their Gaussian values ( 0 and 3 , respectively) at all depths [34]. The small deviations of skewness and kurtosis above their Gaussian values indicates that the dissipation distributions have slightly wider tails and a tendency towards larger values than a Gaussian distribution. Both statistics vary systematically with the depth, with the skewness switching sign in the surface $500 \mathrm{~m}$ (Fig. 1). QGPE dissipation is even closer to being log-normally distributed than the KE dissipation (gray versus black lines), consistent with the expectation that QGPE cascades dominate in this simulation. Enstrophy dissipation statistics (not shown) are similar to those of QGPE dissipation.

To demonstrate the robustness of log-normal dissipation, another season and two other simulations are shown in Fig. 3. The simulations differ only in their parameterization of $\mathbf{F}_{\mathbf{H}}$. The harmonic dissipation using QG Leith viscosity (black curve) [17] and 2D Leith viscosity (blue curve) [30] as well as a biharmonic dissipation with resolution-dependent viscosity (red curve) [18] all show nearly log-normal distributions. A more general discussion of these simulations is in Ref. [19]. Log-normality is also robust across seasons (compare the black profiles).

Previous work has shown that dissipation statistics in 3D turbulence cannot be precisely log-normal [22,35]. More accurate multifractal models for 3D turbulence are able to provide better consistency and predict higher moments $[21,31,36]$. Log-normality is a good approximation for dissipation statistics in a global ocean model, but there are systematic variations from log-normality which may improve through multifractal models or greater precision connecting the dissipation statistics and dynamics [37].

Conclusions and significance.-The dissipation of kinetic energy by horizontal friction follows a log-normal distribution in high-resolution global ocean models. This distribution is accurate for both global and regional statistics and for a range of common horizontal friction parameterizations. The mean and variance of the distribution vary with the depth, size, season, and location of the chosen region. On a global scale, departures from lognormality are small and depend systematically on the depth. A more detailed description of dissipation intermittency, such as multifractal models, would have to account for these variations of ocean turbulence properties.

The present results have implications for a range of physicists and oceanographers. For example, the downscale cascade of energy through the ocean submesoscale has been inferred through localized in situ observations [15] and regional numerical models [38-40], but we have shown that most of the dissipation at the mesoscale occurs in a small number of high-dissipation locations due to the log-normal distribution (90\% of dissipation at a given depth occurs in about $10 \%$ of the world ocean). This distribution presents challenges when extrapolating regional turbulence observations to global or basin-wide statistics, where it is common to assume normal statistics (e.g., [41]).

The statistics of dissipation importantly constrain subgrid schemes in ocean models. In particular, many models use stochastic noise to approximate chaotic subgrid processes. If these stochastic schemes aim to capture the lognormal statistics of KE and QGPE dissipation, our results suggest that the noise applied should be dominantly multiplicative [42-44] (where log-normal distributions are common) rather than additive $[45,46]$ (where normal distributions are common), unless the additive noise has specific properties [47]. If the numerics are such that there are more modes capable of being dissipated than capable of 
causing fluxes (in Ref. [48], this ratio is nearly $3 / 2$ ), then the combination of dissipation and stochastic variability has some limited freedom. The present results suggest that an important ex post facto check of subgrid schemes is the lognormality of dissipation and flux statistics.

The authors acknowledge support from the National Key Research Program of China (2017YFA0604100), NSF OCE-1350795, and ONR N00014-17-1-2963. Professors McWilliams and Marston provided helpful advice.

[1] R. Ferrari and C. Wunsch, Annu. Rev. Fluid Mech. 41, 253 (2009).

[2] B. Fox-Kemper, S. Bachman, B. Pearson, and S. Reckinger, CLIVAR Exchanges 19, 42 (2014).

[3] G. D. Egbert and R. D. Ray, J. Geophys. Res. 106, 22475 (2001).

[4] A. Sen, R. B. Scott, and B. K. Arbic, Geophys. Res. Lett. 35, L09606 (2008).

[5] A. Bracco, J. von Hardenberg, A. Provenzale, J. B. Weiss, and J. C. McWilliams, Phys. Rev. Lett. 92, 084501 (2004).

[6] D. B. Chelton, R. A. Deszoeke, and M. G. Schlax, J. Phys. Oceanogr. 28, 433 (1998).

[7] R. Hallberg, Ocean Modelling 72, 92 (2013).

[8] R. H. Kraichnan, Phys. Fluids 10, 1417 (1967).

[9] J. G. Charney, J. Atmos. Sci. 28, 1087 (1971).

[10] A. N. Kolmogorov, Dokl. Akad. Nauk SSSR 32, 16 (1941).

[11] K. Smith, G. Boccaletti, C. Henning, I. Marinov, C. Tam, I. Held, and G. Vallis, J. Fluid Mech. 469, 13 (2002).

[12] M. J. Molemaker, J. C. McWilliams, and X. Capet, J. Fluid Mech. 654, 35 (2010).

[13] A. Pouquet and R. Marino, Phys. Rev. Lett. 111, 234501 (2013).

[14] R. Marino, A. Pouquet, and D. Rosenberg, Phys. Rev. Lett. 114, 114504 (2015).

[15] A. C. Poje, T. M. Özgökmen, D. J. Bogucki, and A. Kirwan, Phys. Fluids 29, 020701 (2017).

[16] T. Uchida, R. Abernathey, and S. Smith, Ocean Modelling 118, 41 (2017).

[17] S. D. Bachman, B. Fox-Kemper, and B. Pearson, J. Geophys. Res. 122, 1529 (2017).

[18] M. E. Maltrud and J. L. McClean, Ocean Modelling 8, 31 (2005).

[19] B. Pearson, B. Fox-Kemper, S. Bachman, and F. Bryan, Ocean Modelling 115, 42.

[20] A. Yaglom, Sov. Phys. Dokl. 11, 26 (1966).

[21] B. B. Mandelbrot, J. Fluid Mech. 62, 331 (1974).

[22] A. Vincent and M. Meneguzzi, J. Fluid Mech. 225, 1 (1991).
[23] G. Boffetta, A. Celani, and M. Vergassola, Phys. Rev. E 61, R29 (2000).

[24] M. K. Rivera, W. B. Daniel, S. Y. Chen, and R. E. Ecke, Phys. Rev. Lett. 90, 104502 (2003).

[25] S. Chen, R. E. Ecke, G. L. Eyink, X. Wang, and Z. Xiao, Phys. Rev. Lett. 91, 214501 (2003).

[26] Y.-K. Tsang, E. Ott, T. M. Antonsen, Jr., and P. N. Guzdar, Phys. Rev. E 71, 066313 (2005).

[27] B. K. Shivamoggi, Ann. Phys. (Amsterdam) 323, 444 (2008).

[28] L. Fang and N. T. Ouellette, Phys. Rev. Lett. 117, 104501 (2016).

[29] R. Smith et al., Technical Report No. LAUR-10-01853, Los Alamos National Laboratory, 2010.

[30] B. Fox-Kemper and D. Menemenlis, Geophysical monograph 177, 319 (2008).

[31] C. Meneveau and K. Sreenivasan, J. Fluid Mech. 224, 429 (1991).

[32] J. C. McWilliams, J. Atmos. Sci. 42, 1773 (1985).

[33] J. Callies and R. Ferrari, J. Phys. Oceanogr. 43, 2456 (2013).

[34] C. R. Chu, M. B. Parlange, G. G. Katul, and J. D. Albertson, Water Resour. Res. 32, 1681 (1996).

[35] R. H. Kraichnan, J. Fluid Mech. 62, 305 (1974).

[36] U. Frisch, Turbulence (Cambridge University Press, 1995).

[37] N. Peters, J. Boschung, M. Gauding, J. H. Goebbert, R. J. Hill, and H. Pitsch, J. Fluid Mech. 803, 250 (2016).

[38] X. Capet, J.C. McWilliams, M. J. Molemaker, and A. Shchepetkin, J. Phys. Oceanogr. 38, 29 (2008).

[39] X. Capet, J. C. McWilliams, M. J. Molemaker, and A. Shchepetkin, J. Phys. Oceanogr. 38, 2256 (2008).

[40] M. J. Molemaker, J. C. McWilliams, and X. Capet, J. Fluid Mech. 654, 35 (2010).

[41] C. J. Wright, R. B. Scott, D. Furnival, P. Ailliot, and F. Vermet, J. Phys. Oceanogr. 43, 402 (2013).

[42] T. Palmer, R. Buizza, F. Doblas-Reyes, T. Jung, M. Leutbecher, G. Shutts, M. Steinheimer, and A. Weisheimer, ECMWF Tech. Memo 598, 2009.

[43] A. Debussche, N. Glatt-Holtz, R. Temam, and M. Ziane, Nonlinearity 25, 2093 (2012).

[44] M. Andrejczuk, F. Cooper, S. Juricke, T. Palmer, A. Weisheimer, and L. Zanna, Mon. Weather Rev. 144, 1867 (2016).

[45] R. Zia, J. B. Weiss, D. Mandal, and B. Fox-Kemper, J. Phys. Conf. Ser. 750, 012003 (2016).

[46] M. F. Jansen and I. M. Held, Ocean Modelling 80, 36 (2014).

[47] H. Mouri, Phys. Rev. E 88, 042124 (2013).

[48] S. A. Orszag, J. Atmos. Sci. 28, 1074 (1971). 